Fecha de recepción: marzo 2010 Fecha de aceptación: marzo 2011 Versión final: septiembre 2013

\section{Marketing gastronómico. La experiencia de convertir el momento del consumo en un recuerdo memorable}

Sandra Cabrera *

Resumen: Indicadores económicos, expertos y el mismo hombre común, nos hablan del crecimiento de los negocios en el rubro de comidas. Lo que se llamó en los 90 la "revolución gourmet" cobró fuerza de la mano de una tendencia a nivel mundial que revalorizó los placeres de la buena mesa.

El marketing de experiencias, que ha cobrado cada vez mas fuerza en el terreno de la mercadotecnia, es un concepto fácilmente aplicable al ámbito gastronómico. La implicación emocional y la creación de experiencias, son herramientas importantes tanto para lograr la satisfacción así como la fidelización del cliente. Desde una mirada reflexiva, es fundamental tener en claro que nos lo propongamos o no, a través de la propuesta gastronómica estamos produciendo una experiencia en el cliente. Esto nos lleva a reflexionar que cuanto más conscientes seamos en la propuesta, mayor valor podremos brindarle al cliente y superar sus expectativas.

A continuación analizaremos las motivaciones que llevan a un comensal a querer vivir una experiencia gastronómica.

A partir de estos conceptos desarrollaremos las áreas a utilizar desde la perspectiva del marketing gastronómico, para crear valor en la propuesta a través de estrategias centradas en la búsqueda de la implicancia emocional y/o sensorial de los clientes y así obtener experiencias únicas y memorables.

Palabras claves: competitividad - emprendedores - estrategia - fidelización - franchising - gastronomía- innovación - marketing - marketing de experiencias - referidos - satisfacción del cliente - servicio - valor.

[Resúmenes en inglés y portugués en la página 174]

(*) Contadora Pública (UBA) con Maestría en Dirección Estratégica (UB). Capacitadora y asesora independiente. Se especializa en Pymes y microempresas. Es docente de la Facultad de Diseño y Comunicación de la Universidad de Palermo en el área de Negocios y del Colegio de Cocineros Gato Dumas, entre otras instituciones.

\title{
Introducción.
}

\section{Análisis del sector gastronómico}

Desde el año 2002 hasta la actualidad, Argentina sufrió un período de cambio, donde la economía del país se vio altamente influenciada por la devaluación de la moneda. En este escenario, algunos sectores de la industria sufrieron drásticas pérdidas, pero otros se vieron favorecidos 
por la depreciación de la moneda. Este fue el caso del sector turístico, ya que la llegada de extranjeros al país se incrementó notablemente así como el consumo que dicha actividad trae aparejado, entre ellos en el sector gastronómico.

De acuerdo a sondeos de la revista Franchising Magazine Bliman, J.D. (2009) La hotelería y la gastronomía ocupan el $10^{\circ}$ lugar dentro de los sectores en los que se clasifican la actividad económica en la Argentina.

Asimismo, la actividad gastronómica es sumamente relevante en aquellas áreas donde se desarrolla la mayor parte de las actividades económicas y financieras en el PBI total del país. Esto significa que en las provincias mas importantes, económicamente hablando, como la Capital Federal, Córdoba y Santa Fe, la actividad gastronómica representa mas del 70\% de la actividad. La importante inversión corporativa en los últimos años del sector, continúa siendo por la fuerte presencia de las Pymes, muchas de ellas en proceso de modernización.

La construcción de complejos turísticos y ampliación de establecimientos hoteleros (con propuesta gastronómica incluida), así como la apertura de nuevos locales gastronómicos es constante en los últimos años.

Sumado al crecimiento turístico, algunas características de los argentinos ejemplifican el por qué de esta creciente demanda. Los argentinos pasamos mucho tiempo en los bares; ya sea para reuniones de negocios o simplemente conversar con amigos; lo que conjuntamente con la llegada del estruendoso Starbucks ha ocasionado la apertura, profesionalización y actualización de los cafés o bares, así como la diversificación e innovación en sus productos. Por otra parte, al argentino, le gusta pasar buenos momentos en lugares agradables, y la salida a cenar, se ha convertido en uno de los entretenimientos elegidos por un amplio sector. Si bien la elección en materia de comidas del argentino es bastante tradicional, en los últimos años se ha diversificado debido a la incursión de la gastronomía temática en el mercado. No hace falta más que dar un paseo por los polos gastronómicos como Puerto Madero o Palermo Hollywood, para ver que la oferta es muy amplia y podemos encontrar desde sushi, comida árabe, restaurantes griegos, pasando por propuestas temáticas de espacios ambientados con elementos de polo o comida erótica, hasta nuestras típicas parrillas, o restaurantes de pastas, y las populares pizzerías.

Como observamos, el mercado gastronómico muestra una posición saludable dentro del sector de los servicios, y muestra de ello, son algunos datos vinculados con los recursos humanos y el mercado inmobiliario.

La consultora Adecco Argentina, creó una división especializada de Hotelería y Catering, que cuenta con personal calificado que brindan sus servicios las 24 horas, los 365 días del año, cubriendo reemplazos instantáneos así como posiciones a largo plazo.

Por otra parte, en el mercado inmobiliario observamos que han crecido notablemente los alquileres de locales habilitados con fines gastronómicos, lo que ha llevado al incremento de los mismos en las zonas de moda, como Palermo Hollywood. Esto como contrapartida produjo que estos polos gastronómicos se amplíen mas allá de sus fronteras, o bien, den surgimiento a nuevas zonas donde no se concentraban bares o restaurantes, como Villa Urquiza, Saavedra o Devoto, logrando que un porcentaje de la población no se traslade a las zonas de moda.

Por último en cuanto a indicadores del desarrollo gastronómico, incluiremos al crecimiento de las franquicias en el sector. El franchising sigue creciendo en Argentina y en el mundo debido a que es el sistema de expansión comercial mas utilizado por las empresas sobretodo las orienta- 
das al comercio minorista o retail. Más de un tercio de las empresas que otorgan franquicias en nuestro país pertenecen al sector gastronómico. Los motivos de la participación del sector en este sistema son varios; entre ellos, han podido representar un equilibrio entre las necesidades de franquiciantes y franquiciados, las prestaciones que se brindan mutuamente, así como ha quedado demostrado, ser una actividad sustentable en el tiempo si es desarrollado profesionalmente. Como dijimos anteriormente, el sector gastronómico es uno de los más dinámicos y sensibles a los vaivenes económicos y es así como para el empresario que desee entrar en la industria el hecho que el $67 \%$ de los participantes de nuevos negocios fracase es un dato alarmante y poco alentador. Cabe destacar la importancia, para el emprendedor, de analizar las causas de fracaso de los restaurants. Ubicándose, dentro de los primeros factores los siguientes aspectos que se relacionan directamente con nuestro tema a desarrollar:

\section{Errada concepción del concepto}

El concepto es todo, en el proyecto, es su identidad, la esencia de su propuesta. Debe ser el resultado de la sinergia entre la localización, el estilo del menú, el target, la ambientación, y el tipo de servicio. El concepto surge de una idea, y debe plasmarse y tomar cuerpo a través de las distintas áreas que componen el negocio. Seguramente el concepto surge de la mente del emprendedor, pero debe ser llevado a la práctica a través del equipo gerencial, con lo cual, si no tenemos una clara definición del concepto de negocio, seguramente nos veremos en la dificultad de transmitirlo, lo que nos llevaría a un fracaso asegurado

\section{Nombre incorrecto}

El nombre cumple un papel importantísimo, en la identificación de la personalidad del restaurante. El nombre debe ofrecer un adelanto acerca de las características propias de su cocina y ambientación. Tengamos en cuenta que el nombre le da identidad al restaurante, por lo cual, si el nombre es equivocado, estamos enviando un mensaje equivocado, que solo produce confusión en los clientes potenciales.

\section{Duplicado de Ideas}

Muchas veces se toma el mero hecho de copiar como una llave para el éxito. Pero tengamos en cuenta que debemos analizar las condiciones del entorno, target, capacidad financiera y el team gerencial, antes de comenzar. Generalmente se copian algunos aspectos pero se evalúa si pueden tener éxito cambiando de management, localización, menú, etc. La falta de creatividad, la calidad no cuidada del servicio, y la no diferenciación, solo nos conducen al fracaso.

\section{La importancia de la estrategia de marketing}

De acuerdo a los aspectos desarrollados en el apartado anterior, podemos concluir que el sector gastronómico se ha constituido en un área de creciente competitividad y de un vertiginoso desarrollo de nuevos negocios y en donde los competidores actuales, incrementan su oferta de bienes y servicios. Lo que consecuentemente torna vital tomar medidas para generar propuestas innovadoras y competitivas, para lograr la estabilidad de las empresas en el mercado. 
Dentro de este contexto, es fundamental desarrollar estrategias de marketing para, por una parte, aprovechar este despertar a los placeres culinarios, así como para asegurar la continuidad del negocio, y la sostenibilidad de la rentabilidad.

Hace no tantos años que el marketing ha adquirido un papel privilegiado dentro del área de la restauración. En Europa, se inició el desarrollo del marketing en este sector, desde que el restaurador comprendió la necesidad de fijarse objetivos en términos de beneficio, y de asociarlos a la satisfacción al cliente que se ha logrado.

En palabras del célebre Rochat (2000):

El marketing es el arte de hacer converger las acciones de la empresa de cara a satisfacer mejor las necesidades de su clientela, en el marco de políticas coherentes que buscan optimizar la eficacia global de la empresa de cara a su mercado. (Rochat, 2000, p. 92)

Por otra parte, Cooper, B., Floody, B. \& Mc Neill, G. (2003), nos dicen "el marketing requiere de una comprensión adecuada, de quienes son sus clientes, de donde vienen, por qué están allí y qué es lo que esperan de su restaurante" (Cooper, Floody, \& Mc Neill, 2003, p.178).

Hoy en día todo negocio o emprendimiento gastronómico ha comprendido la necesidad de prestar atención al desarrollo de una estrategia de marketing, ha comprobado que el concepto del negocio, así como una adecuada comunicación del mismo, es tan importante como el producto en sí.

Esto trajo aparejado la necesidad de profesionalizar actividades que antes eran realizadas por el mismo emprendedor sin tener ayuda profesional.

Es así como al modo de ver de Rochat (2000), hay tres aspectos fundamentales a gestionar para lograr la continuidad en un negocio gastronómico:

- Conocer y manejar los costos: no sólo los de las materias primas, sino todos los inherentes a la operación, tal es el caso de los recursos humanos, luz, gas, etc.

- Abordar el aprendizaje de un comportamiento profesional

- La aparición de un nuevo tipo de consumidor

Es en este último aspecto donde el marketing cobra un papel fundamental, ya que con sus herramientas debe prestar especialmente atención a los fenómenos que originan los cambios en los clientes. Las tendencias de los gustos alimentarios y las que conciernen a la higiene, salud y entretenimiento, en particular, influyen fuertemente en la creación de este nuevo tipo de consumidores. Debemos focalizarnos entonces, en anticipar la llegada de estas tendencias y adoptar nuevos métodos para llegar al comensal. 


\section{El marketing de experiencias. Concepto}

El marketing a lo largo de los años ha ido adquiriendo diversos enfoques, desde el marketing centrado en el concepto de producto, que afirmaba que los consumidores favorecerían a los productos de mejor calidad y rendimiento o con características novedosas, pasando por el marketing social, donde se hace foco en proteger e incrementar el bienestar del consumidor y el del conjunto de la sociedad.

Así nos encontramos frente al marketing de experiencias que se enfoca en que el cliente no adquiere productos sino experiencias. Por lo cual, las empresas deben diseñar, y gestionar experiencias positivas, intensas y memorables para captar, retener y fidelizar a los clientes.

Pine II y Gilmore (2002), han estudiado las etapas en la evolución del valor económico, y es así como llegan de una economía agraria a una economía de las experiencias.

Esto no quiere decir que antes no existían las "experiencias" sino que se las consideraba integrantes del servicio. O como dicen Pine II y Gilmore (2002):

... cuando se contrata un servicio, se compra un conjunto de actividades intangibles, mientras que, en una experiencia, el cliente paga por pasar el tiempo disfrutando de esa serie de eventos memorables que la empresa le ofrece a cambio de comprometerlo personalmente. (Pine II y Gilmore, 2002, p. 8)

A continuación analizaremos la progresión en el valor económico para los autores, ya que señalan que son cuatro los tipos de economías analizadas.

1. Economía Agraria: commodities o productos primarios.

Es cuando nos referimos a los productos que se venden directamente provenientes de la naturaleza, los que prácticamente no se diferencian. Actualmente su demanda se ha reducido ya que los clientes cada vez requieren menos de los commodities.

2. Economía Industrial: productos o bienes de consumo.

En esta economía se le incorpora valor al bien a través de la industrialización de los mismos; y es con la innovación en los procesos, que se logra mayor diferenciación.

\section{Economía de Servicios.}

En una primer etapa, los fabricantes sumaron servicios a los productos como una forma de mejorarlos, pero con el tiempo se dieron cuenta que los clientes valoraban tanto a los servicios que podían cobrarlos por separado, entonces, pasaron a customizarlos a pedido de los clientes.

\section{Economía de las Experiencias.}

Pine II y Gilmore (2002): "las experiencias son memorables, generan sensaciones personales, emocionales, físicas, intelectuales, incluso espirituales. Por lo tanto, nunca habrá dos experiencias iguales, ya que cada presentación de la experiencia entra en interacción con el estado mental del individuo antes de vivirla". (Pine II y Gilmore, 2002, pag. 9). 
Las empresas deben analizar cuales son las exigencias de sus clientes, anticiparse a sus requerimientos, para incorporar dichas señales en sus propuestas. Es necesario tratar de entender las expectativas y percepciones de los clientes ligadas a las experiencias. Así como entender que para generar impresiones hay que incorporar claves que confirmen la naturaleza de la experiencia deseada.

La pregunta es cuales son las vías para crear experiencias en el ámbito gastronómico, y este punto es el que desarrollaremos a continuación.

\section{El camino hacia una experiencia memorable}

Comenzaremos por analizar cuales son las motivaciones que nos llevan a vivir una experiencia gastronómica. Al respecto, Rochat (2000) subraya que de acuerdo a estudios realizados existen varias motivaciones relacionadas con la búsqueda de satisfacciones alimentarias, sintetizando en cuatro las que considera principales:

1. La motivación que busca la seguridad: dentro de este tipo encontramos aquellos ejemplos donde premia la necesidad de encontrar un sitio seguro; aquellos comensales que necesitan fundamentalmente asegurarse de estar en un lugar donde se les garantice la higiene en el local, así como el cuidado en la manipulación de los alimentos. Podemos incorporar dentro de este grupo, los que privilegian los productos orgánicos, o la cocina natural: es decir, los que efectúan su selección desde la búsqueda del lado sano y natural de la alimentación.

2. La motivación relacionada con las necesidades sociales: Aquí el privilegio lo tiene la necesidad de comunicación, un ámbito donde se faciliten los contactos sociales ya sea con el grupo que uno ha asistido, o la interacción con gente que no conocemos.

3. La motivación relacionada con las necesidades culturales: la gastronomía es una muestra de la cultura de un país o de una región. Esta muestra no pasa simplemente por la propuesta de los platos, sino por la calidad en la recepción, calidad del personal, y la ambientación.

4. La motivación hedonista que afecta a los placeres físicos: esta motivación es la que se convirtió en la más importante desde el punto de vista del marketing.

Rochat (2000), comenta que "el consumidor va en busca de los placeres que se captan por medio de los cinco sentidos, de los placeres gustativos mas diferenciados" (Rochat, 2000, p.96). Este tipo de motivación va variando de acuerdo a los consumidores, pero involucra a todo el universo de los clientes dependiendo de su edad, cultura, gustos, nivel socioeconómico, etc.; pero todos en mayor o menor medida se acercan a la gastronomía por esta motivación hedonista.

De lo que podemos concluir que no es producto del azar que desde hace algunos años haya cobrado cada vez más interés en el ámbito del marketing la idea del marketing de experiencias en gastronomía.

La gastronomía es uno de los ámbitos por excelencia en los que es fácilmente aplicable el concepto de marketing de experiencias. 
La oferta y generación de experiencias de consumo únicas e irrepetibles para los clientes, se ha convertido en un factor clave a la hora de ganar un lugar dentro de este mercado marcadamente competitivo. Sabemos que tanto la fidelización del cliente así como la referenciación positiva son fundamentales para sostener una propuesta gastronómica a largo plazo. Una herramienta importante tanto para lograr que el cliente vuelva o que nos recomiende a sus conocidos, puede estar dada por convertir el momento del consumo en una experiencia memorable que supere las expectativas del comensal.

Es por eso que es importante que manejemos un conjunto de políticas y estrategias innovadoras centradas en la búsqueda de una nueva fuente de ventajas competitivas en la cadena de valor del negocio, que se base en la implicancia emocional de los clientes y en la creación de experiencias conectadas a nuestro producto o servicio.

Debemos trabajar en concientizar cuales ideas podemos implementar en el negocio para convertir el momento del comer en una experiencia. Al prestar un servicio gastronómico estamos generando una experiencia determinada ya sea positiva o negativa, con lo cual, cuanto más conscientes seamos de esta situación, mayor provecho podremos obtener de las herramientas como la ambientación, la propuesta gastronómica y la calidad del servicio.

En primer lugar es importante construir una imagen de marca que enfatice la experiencia que los consumidores podrán vivir al comprar o usar el producto o servicio. El típico caso que se utiliza para ejemplificar este aspecto es el de la cadena de cafeterías Starbucks, que logró transformar a un producto clásico como el café en un negocio mundial gracias a una planificación estratégica de marketing experiencial combinada con una fuerte y creativa campaña comunicacional en lo que a posicionamiento se refiere. En principio, la propuesta de valor de la marca parece basarse en tres pilares fundamentales: en primer lugar se basan en un Café Premium; segundo, la ambientación de los locales en la que se trata de conseguir un sentido de comunidad; y tercero, la filosofía de servicio que se enfoca en la intimidad con el cliente. El objetivo concreto de la marca es convertirse en el "tercer hogar", luego de la casa y el trabajo. Así, si nos detenemos a analizar estos factores, llegamos a la conclusión de que Starbucks nos lleva, casi sin darnos cuenta, desde una perspectiva casi totalmente racional (café de muy buena calidad) a una casi totalmente emocional (ambiente y servicio). La empresa es, entonces, un gran ejemplo de cómo los dos hemisferios de casi cualquier modelo de marketing (racional y emocional), se conjugan para ser el top of mind de muchos amantes de este tipo de negocios.

Otra alternativa es generar una experiencia netamente sensorial, guiada por los atributos del producto. Para poder crear experiencias, existen cinco vías:

- A través de las Sensaciones. El concepto es crear un escenario que genere sensaciones que involucren los cinco sentidos de los clientes. Cuanto más se estimule los sentidos, mayor la fuerza de la experiencia para perdurar en la memoria del cliente. En este aspecto hay que resaltar que para que se convierta en un recuerdo positivo, la experiencia sensorial debe responder estrictamente al sentido del tema central. Un ejemplo de propuesta gastronómica donde se aplica esta vía, es en el caso del llamado Dark Dining Project (Proyecto de cena en la oscuridad), cenas organizadas por cientos de restaurantes de Manhattan, en donde los comensales tienen que vendarse los ojos con antifaces especiales que les brindan a sus clientes, y comer sin saber cuáles platos les servirán. Esta tendencia ya llegó a Buenos Aires y la podemos encontrar en "A ciegas con Luz", que funciona en el mismo espacio donde funciona la propuesta de teatro ciego. Un 
caso icónico del concepto de marketing sensorial así como ejemplo de temático es el del restaurant Te mataré Ramirez. Tomando como esencia de su identidad el erotismo, su propuesta es lo suficientemente sólida como para generar una experiencia única y absolutamente memorable. Podemos comenzar con su carta que posee un menú afrodisíaco basado en sabores gourmet mediterráneos con nombres alusivos formulados bajo la forma de verso erótico. Esta carta es acompañada desde la ambientación, con una estética de cabaret con luz tenue sugerente. A su vez, a esta propuesta le suman el servicio adicional de distintas actividades artísticas para los comensales como títeres, teatro, música; siempre relacionados con la temática erótica. Con lo que completan una muy original propuesta desde los pilares de su identidad que marca una singular experiencia en el recuerdo de sus visitantes.

- A través de las Emociones. Se producen sentimientos y emociones en los clientes. Un recurso que se suele utilizar es el de trasladar la cultura y vivencias de otra región en la propuesta gastronómica a través del "tema central". Al decir, de Pine II y Gilmore (2002), "el tema debe alterar el sentido de la realidad del participante, ya sea su percepción del entorno, la imagen que tiene de sí o su ubicación en el tiempo. Debe hacerlo sentir alejado de su realidad diaria, en todo sentido" (p.12). El tradicional bar celta El Kilkenny, recrea parte de la cultura irlandesa y ofrece la más variada selección de la bebida insignia de la región, que es la cerveza. Desde su fachada que es típicamente británica, así como la decoración interior donde predomina la simbología celta y el concepto de contar con cervezas del mundo, lo ha convertido en una oferta temática que fue la precursora de la movida de los after-office en Buenos Aires, así como es la sede indiscutida de la celebración de San Patrick Day o día de San Patricio.

- A través de los Pensamientos. Las situaciones están vinculadas al intelecto y a la creatividad de los clientes. Este es el caso de los denominados Bares Notables de la Ciudad de Buenos Aires. Son considerados patrimonio cultural de los vecinos, ya que son parte de la historia porteña. Son un testimonio vivo de épocas pasadas desde su arquitectura, ambientación; así como el recuerdo, de los acontecimientos o personajes ilustres de nuestra historia o cultura que han pasado por allí.

- A través de las Acciones. Los clientes se involucran físicamente en la situación. Puedo citar como ejemplo, una nueva moda que ya se inició en Europa y que seguramente muy pronto llegará a nuestro país. Se trata de una propuesta en la que se fusionan la escuela de cocina con el restaurante, y que en Francia es liderada por Látelier de Chefs. Se trata de una escuela restaurante, en la que los comensales elaboran sus propias comidas bajo la guía y supervisión de sus chefs.

- A través de las Relaciones. Las situaciones se vinculan con la interacción con otras personas. La propuesta denominada Bio Experimental del restaurante Bio de cocina orgánica, consiste en que organizan los días lunes cenas. Durante esa noche los comensales trabajaran entre todos los presentes en la preparación de los platos que degustarán esa noche, al tiempo que reciben una clase no solo de cocina, sino de las características culinarias de la zona de donde proviene dicho plato desde una óptica vegetariana. 
Cada una de las vías señaladas puede ser utilizada individualmente para crear una determinada experiencia. Además, pueden asociarse dos o más de ellas en una experiencia global e integrada. La combinación de las distintas vías depende de las características de los clientes. De lo expuesto surge que en el actual escenario, el marketing de relaciones es una vía que le permite a la empresa la creación de valor, convirtiéndose en una herramienta que contribuye en la generación de ventajas competitivas; y que es de comprobada efectividad en el campo gastronómico.

\section{Conclusiones}

Comenzamos el artículo con un breve análisis del sector gastronómico, sintetizándolo como un sector con un vertiginoso crecimiento, con una fuerte necesidad de profesionalización para lograr el éxito. Luego resaltamos la importancia de la estrategia de marketing como elemento diferenciador frente a un sector que se torna cada vez mas competitivo. Para llegar a conceptualizar el marketing de experiencias, así, como se fue modificando la progresión en el valor económico. Seguimos con el desarrollo de las motivaciones relacionadas con la búsqueda de satisfacciones alimentarias para, a partir de las mismas, acentuar la importancia del marketing de experiencias y su fácil aplicabilidad en el mundo gastronómico. Por último, nos centralizamos en las vías para poder generar experiencias, a través de sus conceptos, así como su aplicación a través de diferentes propuestas que ejemplifican su utilización.

A lo largo del trabajo el objetivo fue fijar la atención del lector en la importancia de convertir el momento de consumo en el ámbito gastronómico, en un recuerdo memorable. Esperamos que el presente trabajo motive a una mayor investigación sobre el comportamiento del consumidor de servicios gastronómicos, tan necesario para el éxito de los emprendimientos de este tipo; así como a la búsqueda de propuestas innovadoras en la cadena de valor de la propuesta.

\section{Referencias Bibliográficas}

Cooper, B., Floody, B. \& Mc Neill, G. (2003). Como iniciar y administrar un restaurante. Bogotá: Norma Grupo Editorial.

Kotler, P. y Armstrong, G. (2010). Marketing. (12a. ed.). Madrid: Pearson Educación.

[Revisión del libro La economía de la experiencia, por B. J. Pine II y J. H. Gilmore] Book Sumary 3. Revista Gestión, p.7-21.

Rochat, M. (2000). Marketing y Gestión de la Restauración. Barcelona: Gestión 2000.

Bliman, J.D. (2009). Franchising Magazine. Edic. n³ 39. Buenos Aires: New Press Grupo Impresor S.A. 
Summary: Economic indicators, experts and even average individuals, tell us about the growth of businesses in the food category. What was called in the 90's "gourmet revolution" took hold of the hand of a worldwide trend that appreciated the pleasures of good food.

Marketing experience is a concept easily applicable to the field of gastronomy. Emotional involvement and the creation of experiences, are important tools for achieving both satisfaction and customer loyalty. From a thoughtful look, it is essential to be clear that, beyond our intentions, we are producing an experience through the gastronomic proposal. This leads us to think that the more aware we are in the proposal, the more value we can provide the customer and exceed their expectations. We will discuss the motivations that lead a person to live a dining experience. From these concepts, the areas to be used from a marketing perspective Gourmet, to create value in the proposal through strategies focused on the pursuit of emotional implication and / or sensory and get customers unique and memorable experiences be developed.

Keywords: competitiveness - customer satisfaction - entrepreneurs - franchising - gourmet industry - innovation - loyalty - marketing - marketing experience - referrals - service - strategy - value.

Resumo: Indicadores econômicos, expertos e o mesmo homem comum, falam do crescimento dos negócios no rubro de comidas. Aquilo que se chamou nos anos noventa a "revolução gourmet" se acrescentou a partir duma tendência ao nível mundial que re valorizou os prazeres da boa mesa.

O marketing de experiências, que cobrou cada vez mais força no terreno do marketing, é um conceito facilmente aplicável ao âmbito gastronômico. A implicação emocional e a criação de experiências, são ferramentas importantes tanto para lograr a satisfação assim como a fidelização do cliente. Desde uma mirada reflexiva, é fundamental saber que através da proposta gastronômica estamos produzindo uma experiência no cliente. Isto leva a reflexionar que quanto mais conscientes sejamos na proposta, maior valor poderemos dar ao cliente e superar suas expectativas.

A partir destes conceitos se abarcarão as áreas a utilizar desde a perspectiva do marketing gastronômico, para criar valor na proposta através de estratégias centradas na procura da implicância emocional e/ou sensorial dos clientes e assim obter experiências únicas e memoráveis.

Palavras chave: competitividade - empreendedores - estratégia - fidelização - franchising gastronomia - inovação - marketing - marketing de experiências - referidos - satisfação do cliente - serviço - valor. 